\title{
MACROGLOBULINEMIA DE WALDENSTRÖM: CASO CLÍNICO Y ACTUALIZACIÓN
}

\author{
Quirós Quirós, William y Rojas Castrillo, Yaoska \\ Microbiólogos, Laboratorio Clínico, Hospital Dr. Rafael Ángel Calderón Guardia, San José, Costa Rica.
}

Resumen: La Macroglobulinemia de Waldenström es una neoplasia hematológica poco frecuente que se caracteriza por una infiltración medular de linfocitos relacionados clonalmente, que secretan una proteína monoclonal de tipo IgM. Según la clasificación de la Organización Mundial de la Salud, esta entidad corresponde a un Linfoma No Hodgkin de células B, conocido como Linfoma Linfoplasmocítico, que aparece con mayor frecuencia en la población mayor de 65 años y particularmente, en varones caucásicos. Es indispensable realizar un apropiado diagnóstico diferencial entre esta enfermedad y otras entidades similares con componente monoclonal asociado. La etiología es aún desconocida, pero se ha asociado con factores genéticos y familiares. Se presenta el caso clínico de un paciente afecto de Macroglobulinemia de Waldenström sintomático agresivo con cuadro clínico de hemorragia retiniana y crioaglutininas, más otros signos ocasionados por la enfermedad.

Palabras clave: Macroglobulinemia de Waldenström; Linfoma Linfoplasmocítico; Síndrome de hiperviscosidad 


\title{
WALDENSTRÖM MACROGLOBULINEMIA; CASE REPORT AND UPDATE
}

\begin{abstract}
The Waldenström Macroglobulinemia is a rare hematologic malignancy characterized by medullar infiltration of lymphocytes clonally related, which secrete monoclonal IgM protein. According to the World Health Organization classification, this entity corresponds to a B-cell No Hodgkin Linfom known as lymphoplasmacytic lymphoma that occurs most often in people over 65 years and particularly in Caucasian males. It is essential to make a proper differential diagnosis between this disease and other similar entities associated with monoclonal component. The etiology is unknown but has been associated with genetic factors and family. We report a case of a patient with symptomatic Waldenström Macroglobulinemia, which present an aggressive clinical picture of retinal bleeding plus others typical signs caused by the disease.
\end{abstract}

Key words: Waldenström Macroglobulinemia; lymphoplasmacytic lymphoma; Hyperviscosity Syndrome,

\section{INTRODUCCIÓN}

La Macroglobulinemia de Waldenström (MW) fue descrita por primera vez en 1944 por el Doctor Jan Gosta Waldenström. El Dr. Waldenström informó de dos pacientes con sangrados anormales en mucosas, linfadenopatías, anemia y trombocitopenia. Inicialmente, éste asoció ambas sintomatologías con un escenario preliminar de mieloma múltiple (denominado mielomatosis incipiente); pero posteriormente, los nuevos hallazgos clínicos (ausencia de lesiones óseas, médula ósea con infiltrado linfoide, pero pocas células plasmáticas, aumento de proteínas séricas, elevación de la viscosidad sanguínea y la propensión exagerada a sangrados y hemorragias retinianas) demostraron ser características distintivas de una nueva malignidad hematológica poco habitual y diagnosticada como MW $[1,2,3]$.

La MW es una forma indolente de Linfoma No Hodking de células $B$, distinguida por su infiltración medular de células clonales linfoplasmocíticas y su producción monoclonal de IgM. Usualmente, es precedida de una gammapatía monoclonal IgM de significado incierto, que eleva en 46 veces el riesgo relativo con respecto a la población general [4, 5]. Es una entidad esporádica e infrecuente; dentro de las neoplasias hematológicas constituye menos del 2\% del total [6].

\section{REPORTE DE CASO:}

Varón de 66 años de edad que acude al servicio de emergencias del Hospital Calderón Guardia con un cuadro de adinamia, fatiga, pérdida de peso no cuantificada y poliartralgias de 3 meses de evolución. Una semana antes del ingreso presentó otalgias, mareos, debilidad e hiporreflexia en miembro superior izquierdo, acompañado de dolor articular focalizado en las muñecas.

La exploración física inicial fue negativa, pero en los estudios preliminares de laboratorio se evidencia una anemia franca (hemoglobina: 6,9 $\mathrm{g} / \mathrm{dL}$ ) con microcitos, macrocitos e hipocromía y fenómeno de Rouleaux asociado a una leucocitosis $(16500 / \mu \mathrm{L})$ con predominio de linfocitos. Adicionalmente se reporta la presencia de $6 \%$ de linfocitos atípicos y $9 \%$ de células que se describen con escaso citoplasma y cromatina fina por lo que el paciente es ingresado para observación y valoración (Figura 1) y (Figura 2). 


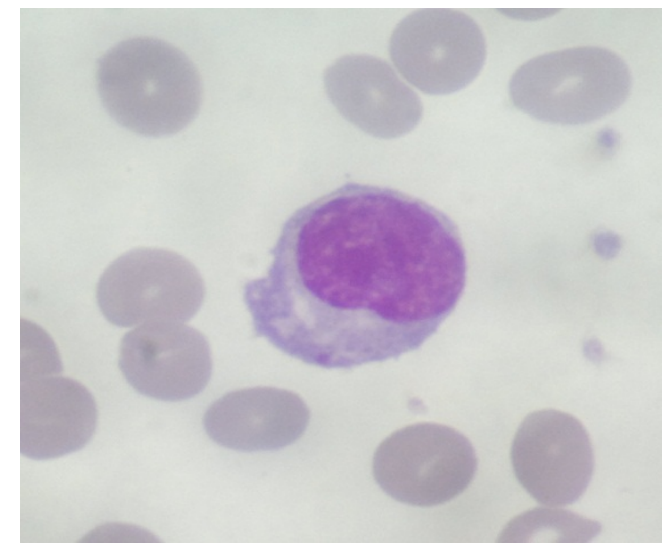

Figura 1. Extendido de sangre periférica mostrando detalle de células linfoplasmacitoides. (Wright x1000).

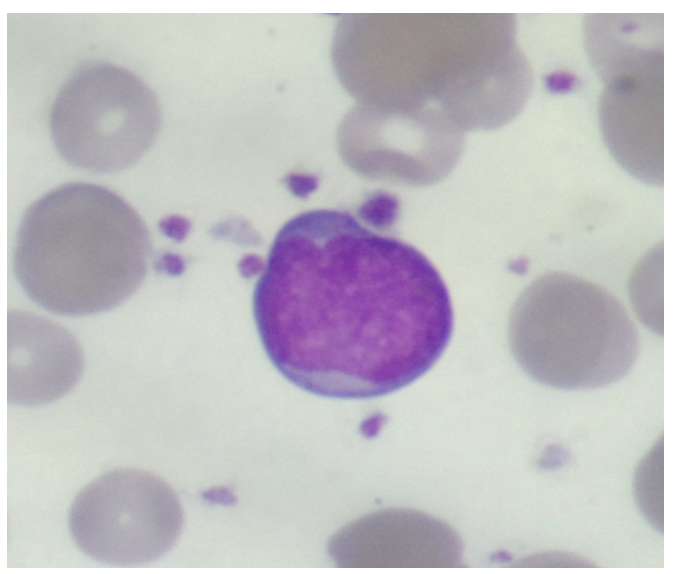

Figura 2. Extendido de sangre periférica mostrando detalle de células linfoplasmacitoide. (Wright x1000).

Las muestras de sangre periférica para los estudios hematológicos fueron obtenidas mediante el procedimiento estandarizado de punción venosa del NCCLS (National Committee for Clinical Laboratory Standars) utilizando tubos al vacío tipo Vacutainer® con EDTA y procesadas en el analizador automatizado Sysmex XE-2100 [7].

La realización de las extensiones de sangre periférica y las tinciones se hicieron de la manera convencional de acuerdo con la recomendación del ICSH (International Committee for Standarization in Hematology) utilizando colorante de Wright y dentro de las dos horas posteriores a su obtención [8].
En los análisis bioquímicos se encontró alteración de la función renal, hiperproteinemia con hipoalbuminemia, elevación de la velocidad de eritrosedimentación y de la proteína C reactiva. El paciente persiste con poliartralgias, mialgias difusas y mareos por lo que se inician estudios por mieloma múltiple. En la exploración física no se observan organomegalias, adenopatías ni edema.

Los estudios por V.D.R.L., Factor Antinúcleo, Citomegalovirus, HIV, Hepatitis A, Hepatitis B, y Hepatitis C fueron negativos.

Se realizó una electroforesis de proteínas donde se confirmó la presencia de una banda gamma monoclonal. La cuantificación de inmunoglobulinas corroboró la presencia de un componente monoclonal con niveles de IgM sérica total de $9370 \mathrm{mg} / \mathrm{dL}$ (40-230 $\mathrm{mg} / \mathrm{dL}$ ), con el resto de las inmunoglobulinas dentro de los parámetros de referencia. En los exámenes inmunohematológicos se reporta una prueba de Coombs directo positivo por anticuerpos de especificidad desconocida y de isotipo IgG (+) e IgM $(+++)$ de amplio rango térmico y no fijadores de complemento.

En el aspirado medular se encontró un infiltrado linfoplasmocitario (Figura 3) por lo que se envía estudio por citometría de flujo.

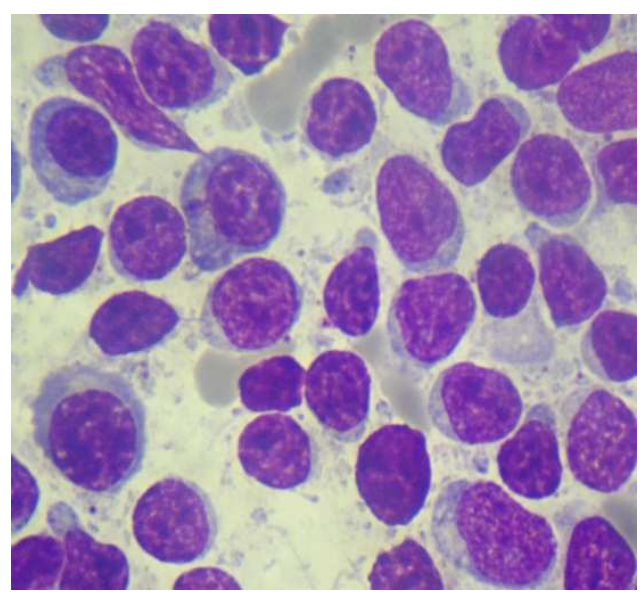

Figura 3. Aspirado de médula ósea mostrando diferentes estados de maduración de linfocitos pequeños, linfoplasmocitos y células plasmáticas. (Wright x1000).

Los estudios de citometría de flujo fueron realizados en el Centro de Referencia Especializado del Hospital Nacional de Niños en San José, Costa Rica con 
las muestras de sangre preservadas con EDTA. Los análisis de médula ósea se realizaron a partir de muestras obtenidas mediante aspirado medular de cresta ilíaca.

El informe inmunofenotípico reveló $57 \%$ de células B patológicas, $40 \%$ de estas células correspondientes a linfocitos B maduros clonales kappa y $17 \%$ con inmunofenotipo de linfoplasmocito B clonal kappa (CD19+, CD20+, CD5-, CD10-, CD23-, CD103- y CD138-) compatibles con la impresión diagnóstica de Macroglobulinemia de Waldenström.

Una exploración radiológica posterior con ecografía de abdomen mostró una esplenomegalia grado IV en el paciente. La serie ósea metastásica fue negativa.

La prueba para viscosidad sérica se reportó en 6.6 (1.4-1.6) por lo que se valoró síndrome de hiperviscosidad. No se encontró déficit neurológico, pero se observó una hemorragia retineana en el ojo derecho por lo que se inicia plasmaféresis con plasma fresco congelado.

Se realizan tres plasmaféresis y se inicia tratamiento con protocolo de quimioterapia R-CHOP (Rituximab, Ciclofosfamida, Doxorubicina, Vincristina, Prednisona). El paciente muestra mejoría clínica hasta el día de hoy con hemograma, bioquímica, velocidad de sedimentación, índice de viscosidad sérica y niveles de IgM dentro de los rangos de referencia.

\section{DISCUSIÓN:}

La MW es una enfermedad derivada de la acumulación, predominantemente en la médula ósea, de linfocitos relacionados clonalmente, células linfoplasmocíticas y células plasmáticas que secretan una proteína IgM monoclonal. Se considera que esta condición corresponde al Linfoma Linfoplasmocítico (LLP) establecido por los sistemas de clasificación de la Organización Mundial de la Salud (0MS, 2008) [9, 10, 11]. La mayoría de los casos de LLP son MW ya que menos del 5\% de los casos corresponden a LLP asociado a IgA, IgG o no secretor. Esta patología representa alrededor del $2 \%$ de todas las malignidades hematológicas en población caucásica y sólo el $6 \%$ de las enfermedades linfoproliferativas de células B $[2,4]$.
La MW es un raro transtorno linfoproliferativo maligno crónico, con una incidencia reportada en Estados Unidos de 3.4 casos/por millón en hombres y 1.7 casos/ por millón en mujeres; la cantidad de casos se elevan conforme aumenta la edad $[12,13]$. La tasa de incidencia es más mayor y casi tres veces más frecuente en caucásicos. La etiología se desconoce y se han relacionado factores genéticos y familiares [14]. Aproximadamente el $20 \%$ de los pacientes son de ascendencia judía de Europa del Este y además, se han reportado numerosos casos de enfermedad familiar, incluyendo presentación multigeneracional de MW y otras enfermedades linfoproliferativas de células B, lo que podría estar relacionado a algunos tipajes de HLA $[12,15]$. El rol de los factores ambientales en MW aún no ha sido esclarecido; sin embargo, la estimulación antigénica crónica producida por infecciones y la exposición a ciertas drogas, podrían estar involucradas en su patogenia $[12,16]$.

Típicamente el cuadro clínico en la MW es de tipo crónico y de lenta aparición. La sintomatología obedece a la infiltración del tumor, a la proteína monoclonal sérica ó a la combinación de ambos eventos. Los signos clínicos atribuibles al crecimiento tumoral son citopenias (la anemia progresiva es la manifestación más común para iniciar el tratamiento), cuadro constitucional más ó menos evidente (astenia, anorexia, pérdida de peso) [13,17]. Además, puede existir infiltración linfomatosa de múltiples órganos: riñón, pulmón, piel, tracto grastrointestinal [18].

La acumulación de inmunoglobulinas monoclonales tiende a producir síndrome de hiperviscosidad, síntomas cardíacos, enfermedad por crioaglutininas y hemorragias producto de la agregación plaquetaria y de la disfunción de los factores de la coagulación $[9,10]$.

La viscosidad sérica está en función de la concentración de IgM, que por su gran tamaño, su disposición pentamérica y su capacidad funcional de anticuerpo, le confiere una gran facilidad para unirse a otros componentes sanguíneos y tisulares, facilitando la formación de complejos y la precipitación en frío de los mismos $[19,20]$.

La sintomatología ligada al síndrome de hiperviscosidad (sangrado espontáneo de mucosas, retinopatías, transtornos neurológicos) suele aparecer cuando el índice de viscosidad sérica es de 5 a 10 veces superior a lo normal, lo cual ocurre entre un $20-30 \%$ de los casos $[13,17,21]$.

Revista electrónica publicada por el Departamento de Farmacología de la Escuela de Medicina de la Universidad de Costa Rica, 2060 San José, Costa Rica. ${ }^{\circledR}$ All rights reserved. Licensed under a Creative Commons Unported License. 
La hiperviscosidad debe ser considerada cuando se tienen valores superiores a 4.4 y debe ser abordada con prontitud, siendo la aféresis terapéutica la alternativa más adecuada $[22,23]$.

En el presente caso, el afectado tuvo MW sintomática, con manifestaciones derivadas de la infiltración tumoral y de la gammapatía monoclonal. Las complicaciones oculares encontradas, son el resultado indirecto de la alta viscosidad sérica. Por tanto, se indica el recambio plasmático como opción terapéutica precoz para el manejo agudo de los trastornos originados por el síndrome de hiperviscosidad, que es muy eficaz dado que la IgM se sintetiza lentamente $\mathrm{y}$ permanece mayormente intravascular [23, 24, 25].

Al paciente descrito se le efectuaron tres procedimientos utilizando plasma fresco congelado como solución de remplazo, éste fluido no es el adecuado para remover la inmunoglobulina anómala en éstos casos. Idealmente, se debe emplear albúmina para efectuar el intercambio, debido a que fundamentalmente no aporta los anticuerpos en remoción; además, no altera la presión oncótica, no aporta mediadores inflamatorios ni riesgo viral; su único inconveniente es su elevado costo económico [23, 26].

En la Macroglobulinemia Primaria la aféresis terapéutica suele ser rápida y efectiva para revertir las complicaciones asociadas a la hiperviscosidad. Las plasmaféresis deben ser diarias hasta que desaparezcan los síntomas y después, deben repetirse cada 1 ó 2 semanas [23]. Paralelamente, es adecuado iniciar con terapia citorreductora; empero, la aféresis puede estar indicada indefinidamente si no hay respuesta a la quimioterapia y continúa la sintomatología [17, 27].

También, es característico en este tipo de neoplasias encontrar al frotis los eritrocitos en pilas de monedas ó "rouleaux" por la paraproteinemia; al mismo tiempo, se observó linfoplasmocitos en sangre periférica, este hallazgo es poco común, debido a que estas células pueden ser confundidas con linfocitos atípicos $[1,11,28]$.

Los criterios diagnósticos más importantes de la MW son la cuantificación de un pico monoclonal en la región gamma, producto del aumento de la IgM, junto con la presencia de un infiltrado linfoplasmocítico en médula ósea con un inmunofenotipo característico $[1,28,29]$. Los estudios de proteínas totales están aumentados debido al incremento del componente $\mathrm{M}$, la electroforesis pone en evidencia la banda cuya identificación corresponde a la IgM clonal. El resto de las inmunoglobulinas policlonales generalmente se encuentran normales ó disminuidas. Además, la IgM puede comportarse como crioaglutinina y dar las pruebas cruzadas ó de antiglobulina directa positivas por unión inespecífica del anticuerpo a los glóbulos rojos, como sucede en éste caso $[20,21,30]$.

Aún en individuos de edad avanzada, la toma del aspirado y/ó biopsia de médula ósea es precisa para el diagnóstico de la MW. El análisis de la muestra medular muestra hipercelularidad y pleomorfirsmo de la línea B dada por linfocitos en diferentes estados de maduración y de ubicación intratrabecular, como linfocitos pequeños, linfoplasmocitos y células plasmáticas; acompañadas de mastocitos aumentados en número [18, 31, 32]. En el estudio inmunofenotípico característicamente, las células neoplásicas $\mathrm{B}$ exhiben ausencia de reactividad para CD5, CD10 y CD23, expresión de marcadores pan-B (CD19, CD20, CD22, CD79a) y de inmunoglobulina de superficie (sIgM+), positividad constante para CD25 y CD54 y reactividad variable para CD38 $[17,31]$.

Es indispensable realizar un apropiado diagnóstico diferencial entre esta enfermedad y otras entidades similares con componente monoclonal IgM asociado como Gammapatía Monoclonal de Significado Incierto, Mieloma Múltiple, Leucemia Linfocítica Crónica, Linfomas No Hodgkin y trastornos relacionados con IgM en ausencia de infiltrado medular por Linfoma Lifoplasmocítico [9, 33, 34].

El reporte del inmunofenotipo es de gran ayuda para distinguirla MW de otros neoplasmas linfoproliferativos crónicos $B$, especialmente de Leucemia Linfocítica Crónica y de algunos Linfoma No Hodgkin con componente monoclonal IgM, como: Linfoma de la Zona Marginal Esplénica (LSZM) y Linfoma de Células del Manto (LCM); puesto que, el cuadro clínico es semejante. En la Leucemia Linfocítica Crónica, los linfocitos son CD5 + y CD23+ (negativos en MW); por lo demás, los Linfomas No Hodgkin se distinguirán por morfología de la biopsia ganglionar y suelen acompañarse de anomalías citogenéticas particulares de cada uno $[5,13,35]$.

Debido a las implicaciones clínicas entre el Mieloma Múltiple (MM) y la Gammapatía Monoclonal de Significado Incierto (GMSI), es relevante su diferenciación; ésta última, se caracteriza por la

Revista electrónica publicada por el Departamento de Farmacología de la Escuela de Medicina de la Universidad de Costa Rica, 2060 San José, Costa Rica. ${ }^{\circledR}$ All rights reserved. Licensed under a Creative Commons Unported License. 
presencia de IgM monoclonal en ausencia de infiltración en médula. Pese a lo anterior, desde hace varios años se ha llegado al consenso de no indicar tratamiento a los pacientes con GMSI ó con MW asintomática (smolderingó indolente), sólo se les efectúa un minucioso seguimiento cada 3-6 meses, ya que éstos permanecen estables durante años [32, 33, 34, 35].

Finalmente, la distinción entre MM por IgM y MW ocurre por el predominio de células plasmáticas neoplásicas en el aspirado óseo del primero; además, de la presencia de lesiones osteolíticas en sacabocados y el dolor óseo; infrecuente en MW (aproximadamente sólo el $2 \%$ de los enfermos muestra compromiso osteolítico difuso) $[18,20]$.

Aún no ha sido definido un algoritmo de tratamiento preciso para MW. En el reporte de caso se utilizó aferésis plasmática para solucionar las complicaciones derivadas de la hiperviscosidad y en conjunto se administró régimen de quimioterapia RCHOP. Éste se encuentra dentro de las opciones de primera línea en MW sintomáticas con comportamiento agresivo; es necesario definir la terapia adecuada para cada paciente, tomando consideraciones individuales que incluyan la presencia de citopenias, la necesidad de control rápido de la enfermedad, la edad y la posibilidad de trasplante autólogo [36,37,38,39].

La anemia en el paciente refleja tanto el involucramiento medular de la enfermedad como el nivel sérico aumentado de la IgM; el valor de hemoglobina se considera un fuerte factor pronóstico adverso si se encuentra por debajo de 9-12 g/dL y se ha relacionado con una menor sobrevida [26]. Algunos otros elementos desfavorables mencionados en la literatura son: niveles altos de $\beta-2$ microglobulina $(>3-$ $3.5 \mathrm{~g} / \mathrm{dL})$ e IgM sérica $(>7,000 \mathrm{mg} / \mathrm{dL})$, presencia de crioglobulinas y trombocitopenia ó leucopenia $[12,14]$.

En conclusión, la MW es una neoplasia con marcada diferenciación plasmocitoide de baja incidencia, de manera característica presenta un componente monoclonal IgM elevado en suero y crecimiento tumoral en medula ósea, los síntomas derivan de ambos fenómenos y en el caso del paciente descrito, la clínica se mostró de manera florida y apoya los hallazgos bioquímicos, morfológicos y de citometría de flujo encontrados. Además, la poca bibliografía existente a nivel nacional sobre esta patología, hace importante su informe y discusión.

\section{REFERENCIAS}

1. Ortiz C. De las células plasmáticas al mieloma múltiple. Una breve perspectivahistórica. Patología 2011; 49 (2): 120-131.

2. Schuster SR, Rajkumar SV, Dispenzieri A, Morice W, Aspitia AM, Ansell S et. al. IgM multiple myeloma: disease definition, prognosis, and differentiation from Waldenstrom'smacroglobulinemia. Am J Hematol 2010; 85 (11): 853-855.

3. Ott G, Balague-Ponz 0,de Leval L,de Jong D,Hasserjian RP, Elenitoba-JohnsonK. Commentary on the WHO classification of tumors of lymphoid tissues (2008): indolent B cell lymphomas. J Hematop 2009; 2 (2): 77-81.

4. Morel P, Merlini G. Risk stratification in Waldenströmmacroglobulinemia. Expert Rev Hematol 2012; 5 (2): 187-199.

5. Stone MJ, Pascual V. Pathophysiology of Waldenström'smacroglobulinemia.

Haematologica2010; 95(3): 359-364.

6. Hivert B, Tamburini J, Vekhoff A,Tournilhac O, Leblond V, Morrel P. Prognostic value of the International Scoring System and response in patients with advanced Waldenströmmacroglobulinemia. Haematologica 2011; 96 (5): 785-788.

7. Ernst DJ, Ballance Lo, Calam RR, McCall R, Smith SS, Szamosi DI, Warunek DJ. National Committee for Clinical Laboratory Standars. Precedures for the Collection of diagnostic blood specimens by venipunture; Approved Standar-Sixth Edition. 2007; H3-A6, Vol. 27 No. 26 Replaces H3-A5 Vol. 23 No. 32. Disponible en: http://www.clsi.org/source/orders/free/H3-a6.pdf.

8. International Committee for Standarization in Haematology (ICSH). ICSH reference method for staining of blood and bone marrow by azure B and eosin Y (Romanowsky stain). Br J Haematol. 1984; 57:707-10

9. Ansell SM, Kyle RA, Craig RB, Fonseca R, Mikhael JR, Morice WG et. al. Diagnosis and Management of WaldenströmMacroglobulinemia: Mayo Stratification of Macroglobulinemia and RiskAdapted Theraphy (mSMART) Guidelines. Mayo ClinProc 2010; 85 (9): 824-833. 
10. Gertz MA. Waldenström Macroglobulinemia: 2012 update on diagnosis, risk stratification, and management. Am. J. Hematol 2012; 87 (5): 504-510.

11. Neparidze N, Dhodapkar MV. Waldenström's Macroglobulinemia: recent advances in biology and theraphy. Clin Adv Hematol Oncol 2009; 7 (10): 677690.

12. Treon S, Hatjiharissi E, Leleu X, Roccaro A, Merlini G. Waldenström Macroglobulinemia/ Lymphoplasmacytic Lymphoma. En: Hoffman R, Benz EJ, Shattil SJ, Furie B, Silberstein LE, McGlave P et. al. Hematology: basic principles and practice. 5 ed. Philadelphia: Churchill Livingstone Elsevier; 2008. p.1413-1425.

13. Vijay A, Gertz MA. Waldenström macroglobulinemia. Blood 2007; 109 (12): 5096-5103.

14. Morel P, Duhamel A, Gobbi P, Dimopoulos MA, Dhodapkar MV, McCoy J et. al. International prognostic scoring system for Waldenstrom macroglobulinemia. Blood 2009; 113 (18): 41634170.

15. Kristinsson SY, Goldin LY, Turesson I, Björkholm M, Landgren 0 . Genetic and immune-related factors in the pathogenesis oflymphoplasmacytic lymphoma/Waldenström's Macroglobulinemia. Clin Lymphoma Myeloma 2009; 9 (1): 23-26.

16. Braggio E, Philipsborn C, Novak A, Hodge L, Ansell S, Fonseca R. Molecular pathogenesis of Waldenstrom's macroglobulinemia. Haematologica 2012; 97 (9): 1281-1290.

17. Treon S. How I treat Waldenström macroglobulinemia. Blood 2009; 114 (12): 23752385.

18. Ghobrial IM, Zhang Y, Liu Y, Ngo H, Azab F, Sacco A, et. al. Targeting the bone marrow in Waldenström Macroglobulinemia. Clin Lymphoma Myeloma Leuk 2011; 11 Suppl 1: 65-69.

19. Leleu X, Xie W, Bagshaw M, Banwait R, Leduc R, Roper $\mathrm{N}$ et. al. The role of serum immunoglobulin free light chain in response and progression in Waldenstrom macroglobulinemia. Clin Cancer Res 2011; 17 (9): 3013-3018.

20. Romaní F, Caudra J, Atencia F y Canelo C. Macroglobulinemia de Waldenström: comunicación de un caso. An Facmed 2008; 69 (2): 112-116.
21. Issa GC, Ghobrial IM, Roccaro AM. Novel agents in Waldenström macroglobulinemia. Clin Investig (Lond) 2011; 1 (6): 815-824.

22. Leleu X, Gay J, Roccaro AM, Moreau AS, Poulain S, Dulery $\mathrm{R}$ et. al. Update on therapeutic options in Waldenström macroglobulinemia. Eur J Haematol 2009; 82 (1): 1-12.

23. Restrepo CA, Márquez E y Sanz MF. Therapeutic plasma exchange: types, techniquesand indications in internal medicine. Acta Med Colomb 2009; 34 (1): 23-32.

24. Hunter ZR, Manning RJ, Hanzis C, Ciccarelli BT, Ioakimidis L, Patterson CJ et. al. IgA and IgG hypogammaglobulinemiain Waldenström's macroglobulinemia. Haematologica 2010; 95 (3): 470-475.

25. Menke MN, Feke GT, McMeel JW, Treon SP. Effect of plasmapheresis on hyperviscosity-related retinopathy and retinal hemodynamics in patients with Waldenstrom's macroglobulinemia. Inves Ophtalmol Vis Sci 2008; 49 (3): 1157-1160.

26. Roccaro AM, Leleu X, Botta S, Burwik N, Vacca A, Russo D et. al. Waldenström's Macroglobulinemia: new therapeutic options. Cancer Theraphy 2008; 6 (1): 227-238.

27. Szczepiorkowski ZM, Winters JL, Bandarenko N, Kim $\mathrm{HC}$, Linenberger ML, Marques MB et al. Guidelines on the use of Therapeutic apheresis in clinical practiceEvidence-based approach from the apheresis applications committee of the American Society for apheresis. J. Clin. Apheresis 2010; 25: 83-177.

28. Olmo J, España A, Idoate MA, Panizo C. Macroglobulinemia de Waldenström asociada a lesiones cutáneas y crioglobulinemia tipo I. Actas Dermosifiliogr 2008; 99 (1): 138-144.

29. Perrot A, Pionneau C, Azar N, Baillou C, Lemoines FM, Leblond $\mathrm{V}$ et al. Waldenstrom's macroglobulinemia harbors a unique proteome where Ku70 is severely underexpressed as compared with other Blymphoproliferative disorders. Blood Cancer Journal 2012; 88 (2).

30. Rudmann SV. Serologic problem-solving: a systematic approach for improved practice. Bethesda: AABB Press; 2005.

31. Konoplev S, Medeiros LJ, Bueso-Ramos CE, Jorgensen JL, Lin P. Immunophenotypic profile of lymphoplasmacytic lymphoma/Waldenström 
macroglobulinemia. Am J Clin Pathol 2005; 124 (3): 414-420.

32. Hodge LS, Novak AJ, Grote DM, Braggio E, Ketterling $R P$, Manske $M K$ et. al. Establishment and characterization of a novel Waldenström macroglobulinemia cell line, MWCL-1. Blood 2011; 117 (12): 190-197.

33. Kristinsson SY, Tang M, Pfeiffer RM, Björkholm M, Goldin LR, Blimark C et. al. Monoclonal gammopathy of undetermined significance and risk of infections: a population-based study. Haematologica 2012; 97 (6):854-858.

34. Shiseki M, Masuda A, Watanabe N, Fujii M, Kimura T, Yoshinaga $\mathrm{K}$ et. al. Development of diffuse large Bcell lymphoma in a patient with Waldenström's macroglobulinemia/ lymphoplasmacytic lymphoma: clonal identity between two B-cell neoplasms. Hematology Reports 2011; 3: 28-31.

35. Morice WG, Chen D, Kurtin PJ, Hanson CA, McPhail ED. Novel immunophenotypic features of marrow lymphoplasmacytic lymphoma and correlation with Waldenström's macroglobulinemia. Mod Pathol2009; 22 (6): 807-816.

36. Sacco A, Issa GC, Zhang Y, Liu Y, Maiso P, Ghobrial IM et. al. Review Epigenetic modifications as key regulators of Waldenstrom's Macroglobulinemia biology. J Hematol Oncol 2010; 3 (38).

37. Leleu X, Soumerai J, Roccaro A, Hatjiharissi E, Hunter $\mathrm{ZR}$, Manning $\mathrm{R}$ et. al. Increased incidence of transformation and myelodysplasia/acute leukemia in patients with Waldenström macroglobulinemia treated with nucleoside analogs. J Clin Oncol 2009; 27 (2): 250-255.

38. Leleu X, Tamburini J, Roccaro A, Morel P, Soumerai J, Lévy $\mathrm{V}$ et. al. Balancing risk versus benefit in the treatment of Waldenström's Macroglobulinemia patients with nucleoside analogue-based therapy. Clin Lymphoma Myeloma 2009; 9 (1): 71-73.

39. Ricci F, Tedeschi A, Morra E. Therapy-Related Myeloid Neoplasms in Chronic Lymphocytic Leukemia and Waldenstrom's Macroglobulinemia. Mediterr J Hematol Infect Dis 2011; 3(1).

\section{INFORMACION DE AUTOR:}

Dr. William Quirós Quirós. Microbiólogo Químico Clínico - Especialista en Hematología Jefe División de Hematología. Laboratorio Clínico Hospital Dr. Rafael Ángel Calderón Guardia San José, Costa Rica.

e-mail: wqquiros@gmail.com 